\title{
Pathogenesis of cardiac ischemia reperfusion injury is associated with CK2a-disturbed mitochondrial homeostasis via suppression of FUNDC1-related mitophagy
}

\author{
Hao Zhou $\left(^{1,2} \cdot\right.$ Pingjun Zhu ${ }^{1} \cdot$ Jin Wang ${ }^{1} \cdot$ Hong Zhu $^{2} \cdot$ Jun Ren $^{2} \cdot$ Yundai Chen ${ }^{1}$
}

Received: 21 November 2017 / Revised: 25 January 2018 / Accepted: 12 February 2018

(c) The Author(s) 2018. This article is published with open access

\begin{abstract}
Disturbed mitochondrial homeostasis contributes to the pathogenesis of cardiac ischemia reperfusion (IR) injury, although the underlying mechanism remains elusive. Here, we demonstrated that casein kinase $2 \alpha$ (CK2 $\alpha)$ was upregulated following acute cardiac IR injury. Increased CK2 $\alpha$ was shown to be instrumental to mitochondrial damage, cardiomyocyte death, infarction area expansion and cardiac dysfunction, whereas cardiac-specific $\mathrm{CK} 2 \alpha$ knockout $\left(\mathrm{CK} 2 \alpha^{C K O}\right)$ mice were protected against IR injury and mitochondrial damage. Functional assay indicated that CK2 $\alpha$ enhanced the phosphorylation (inactivation) of FUN14 domain containing 1 (FUNDC1) via post-transcriptional modification at Ser13, thus effectively inhibiting mitophagy. Defective mitophagy failed to remove damaged mitochondria induced by IR injury, resulting in mitochondrial genome collapse, electron transport chain complex (ETC) inhibition, mitochondrial biogenesis arrest, cardiolipin oxidation, oxidative stress, mPTP opening, mitochondrial debris accumulation and eventually mitochondrial apoptosis. In contrast, loss of CK2 $\alpha$ reversed the FUNDC1-mediated mitophagy, providing a survival advantage to myocardial tissue following IR stress. Interestingly, mice deficient in both CK2 $\alpha$ and FUNDC1 failed to show protection against IR injury and mitochondrial damage through a mechanism possible attributed to lack of mitophagy. Taken together, our results confirmed that $\mathrm{CK} 2 \alpha$ serves as a negative regulator of mitochondrial homeostasis via suppression of FUNDC1required mitophagy, favoring the development of cardiac IR injury.
\end{abstract}

Edited by L. Scorrano

Electronic supplementary material The online version of this article (https://doi.org/10.1038/s41418-018-0086-7) contains supplementary material, which is available to authorized users.

Hao Zhou

zhouhao301@outlook.com

$\triangle$ Jun Ren

jren@uwyo.edu

$\triangle$ Yundai Chen

yundaic@163.com

1 Chinese PLA General Hospital, Medical School of Chinese PLA, Beijing, China

2 Center for Cardiovascular Research and Alternative Medicine, University of Wyoming College of Health Sciences, Laramie, WY 82071, USA

\section{Introduction}

One of the hallmarks of cardiac ischemia reperfusion (IR) injury is the altered architecture and function of mitochondria [1]. In-depth researches from a number of laboratories have validated the importance of myocardial death following mitochondrial damage as the essential molecular basis of cardiac IR injury [2, 3]. Recently, accumulating evidence has depicted an indispensable role of mitophagy in mitochondrial protection [4]. Mitophagy, the mitochondrialspecific analog to autophagy, is considered a beneficial metabolic event and is pivotal to the preservation of mitochondrial quality [5]. Evidence from our group has shown that enhanced mitophagy alleviates mitochondrial oxidative stress, preserves mitochondrial respiratory function, limits mitochondrial debris formation, and blocks caspase 9related apoptosis [3,6-9]. Accordingly, mitophagy is thus proposed to be a mechanistic requirement of cellular survival in cardiac IR injury and could be a promising target to relieve the IR attack [10]. 
A
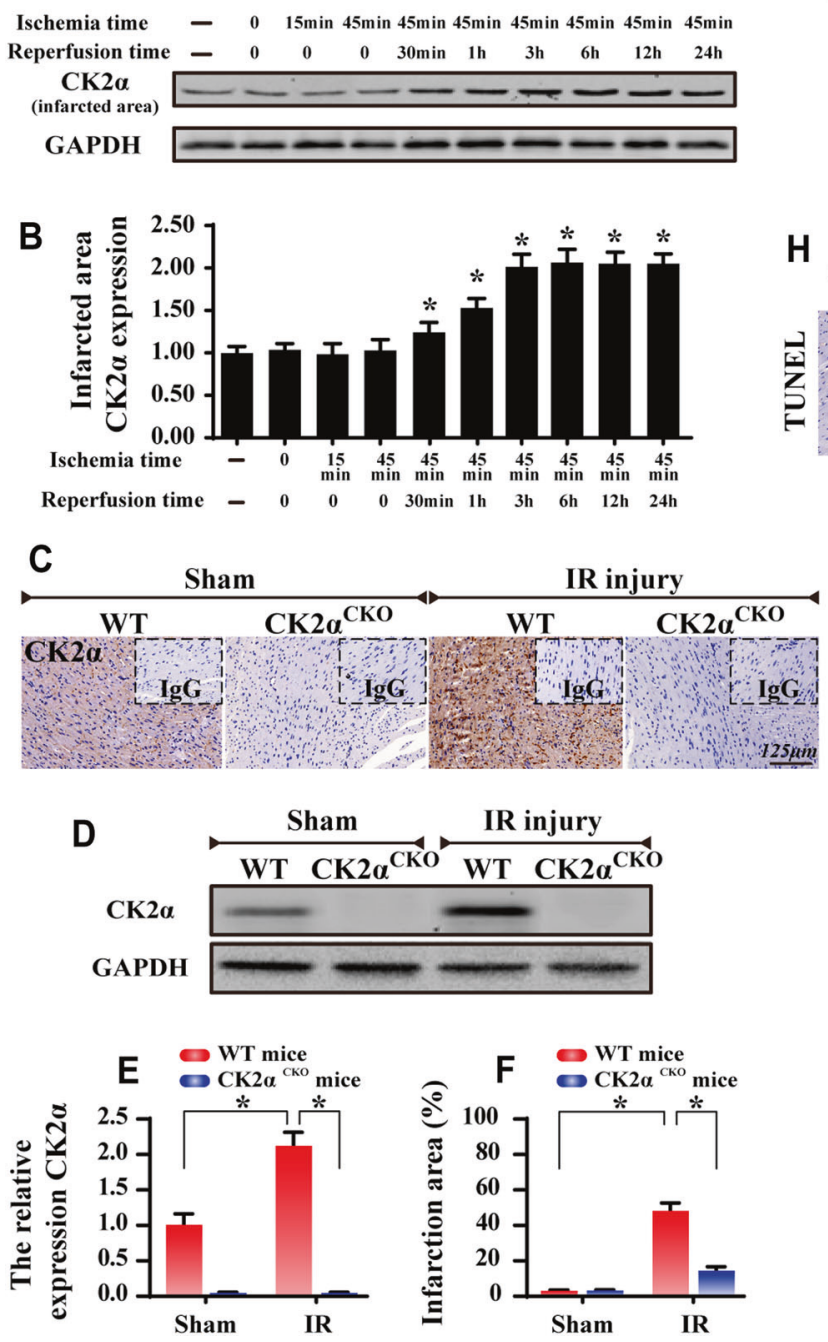

Fig. 1 Upregulation of CK2 $\alpha$ at the infarcted area. WT mice and $\mathrm{CK} 2 \alpha^{C K O}$ mice underwent the 45-min ischemia and 0-24-hour reperfusion (IR injury, $n=6 /$ group). In vitro, 45 -min of hypoxia and 6-hour of reoxygenation (HR) was used to mimic the IR injury. Meanwhile, the loss-of-function assay about $\mathrm{CK} 2 \alpha$ was conducted via siRNA (RNAi-CK2 $\alpha$ ) or control siRNA (RNAi-ctrl) in cardiomyocytes. a CK2 $\alpha$ expression in the infarcted area. b Quantitative analysis of the relative expression of $\mathrm{CK} 2 \alpha$. C. The immunohistochemistry of $\mathrm{CK} 2 \alpha$ in heart. d, e The western blots were used to confirm the CK $2 \alpha$

Mitophagy is regulated through two distinct signaling pathways including receptor-mediated mitophagy and Parkin-dependent mitophagy [11]. The receptor-related components include BCL2/adenovirus E1B $19 \mathrm{kDa}$ protein-interacting protein 3 (Bnip3) and FUN14 domain containing 1 (FUNDC1) [12]. Among which, Bnip3 is a mitochondrial outer membrane protein with biphasic effects [13], first the promotion of mitophagy and later the facilitation of cardiomyocyte death [9]. Similarly, Parkin-related mitophagy also exacerbates cardiomyocyte death in IR injury $[14,15]$. On the other hand, FUNDC1-mediated
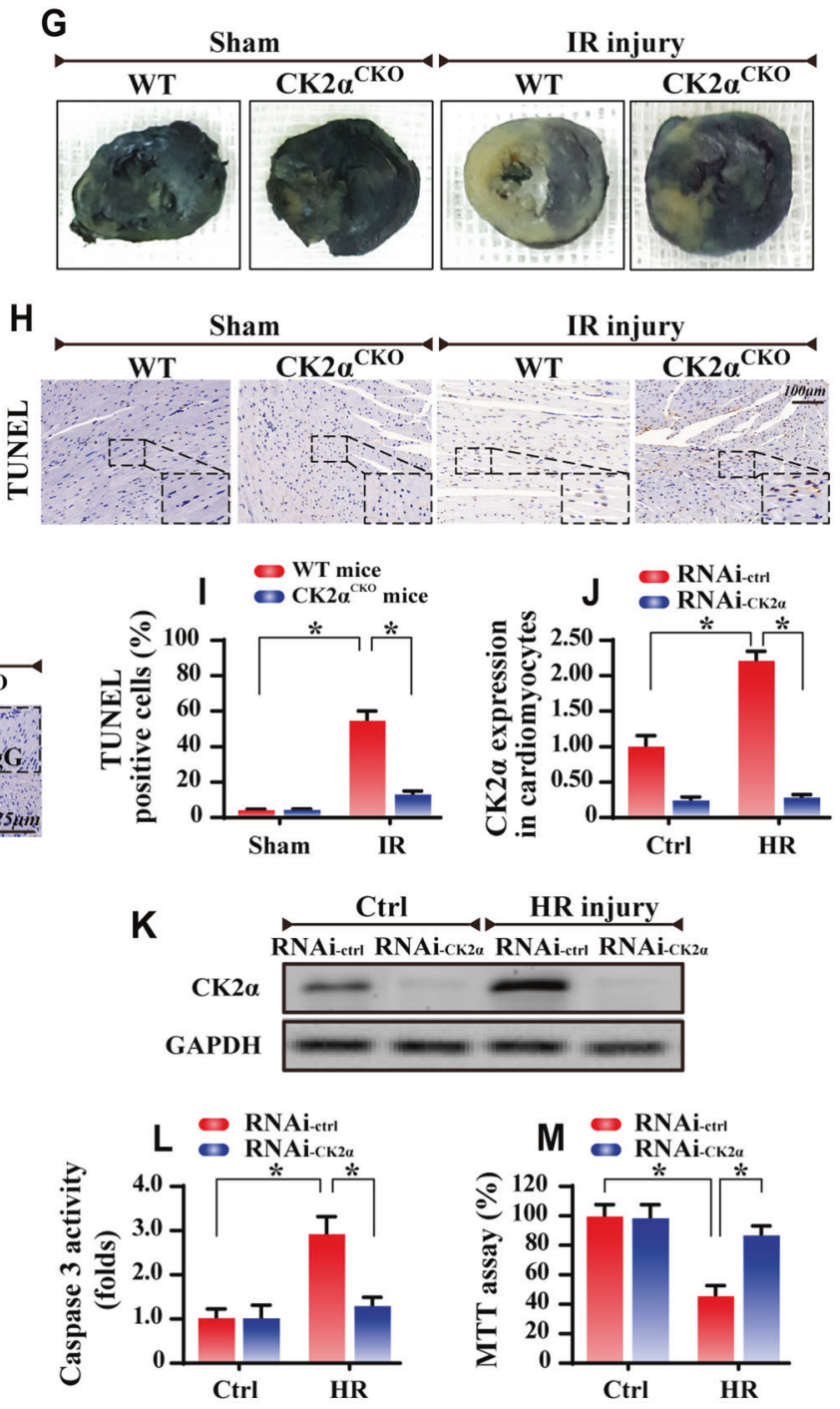

expression in WT mice or CK2 $\alpha^{C K O}$ mice under IR injury. f, $\mathbf{g}$ Representative images of heart sections with TTC and Evans Blue staining of the infarcted area. Bar graph indicates the infarct size. $\mathbf{h}, \mathbf{i}$ TUNEL assay for cellular apoptosis analysis. $\mathbf{j}$, $\mathbf{k}$ The control siRNA (RNAi-ctrl) and CK2 $\alpha$-siRNA (RNAi-CK2 $\alpha$ ) were transfected into cardiomyocytes. The transfection efficiency was confirmed by western blots. 1, $\mathbf{m}$ Caspase 3 activity and MTT assay were used to detect the cellular viability and apoptosis. The data represent the mean $\pm \mathrm{SEM}$. $* P<0.05$

mitophagy is mainly activated early by hypoxia in order to repair damaged mitochondria and offers a survival advantage for cardiomyocytes in IR injury [16, 17]. It is believed that FUNDC1-mediated mitophagy functions in a manner reminiscent of ischemic preconditioning. To this end, different mitophagy adaptors may have disparate impacts on cell fate, and that FUNDC1 may serve as the key mediator turning on protective mitophagy. Structurally, FUNDC1 has two essential phosphorylation sites including Tyr18 and Ser13. Phosphorylated FUNDC1 generates steric hindrance for LC3II binding [18], thus effectively inhibiting 
- WT mice $\rightarrow$ CK2 $\alpha^{\mathrm{CKO}}$ mice

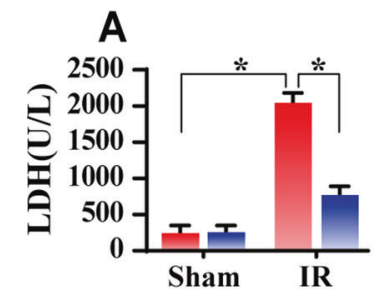

E
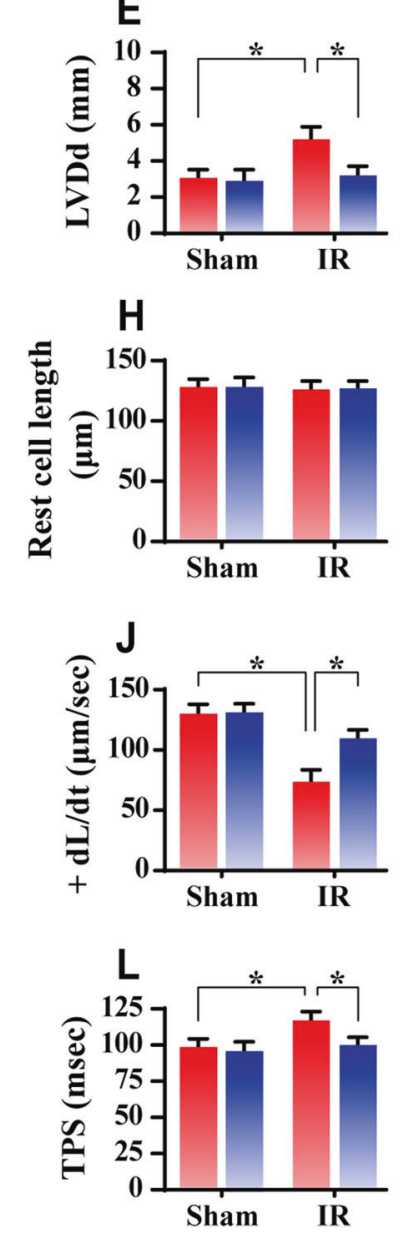

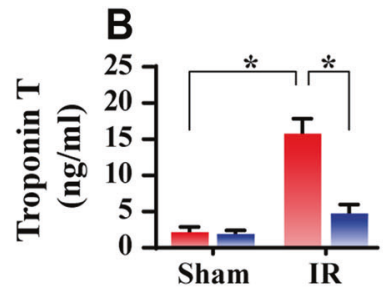

$\mathbf{F}$
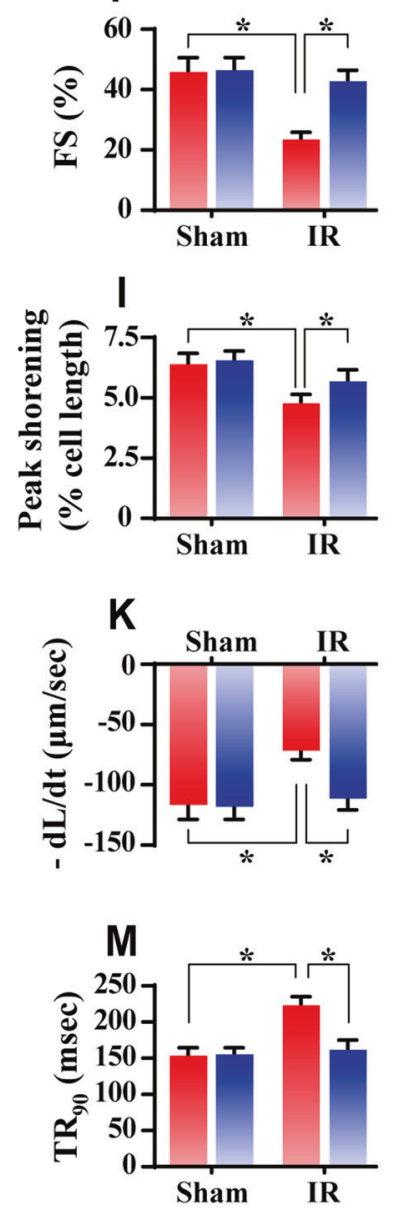
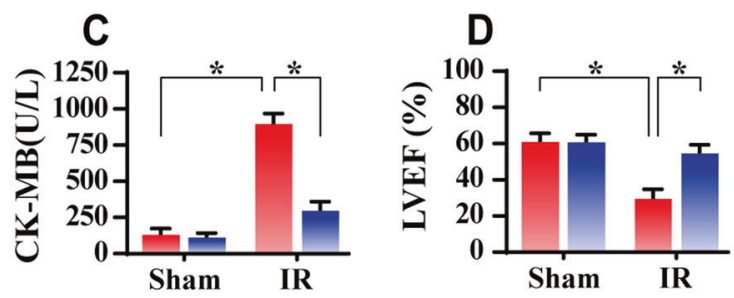

G
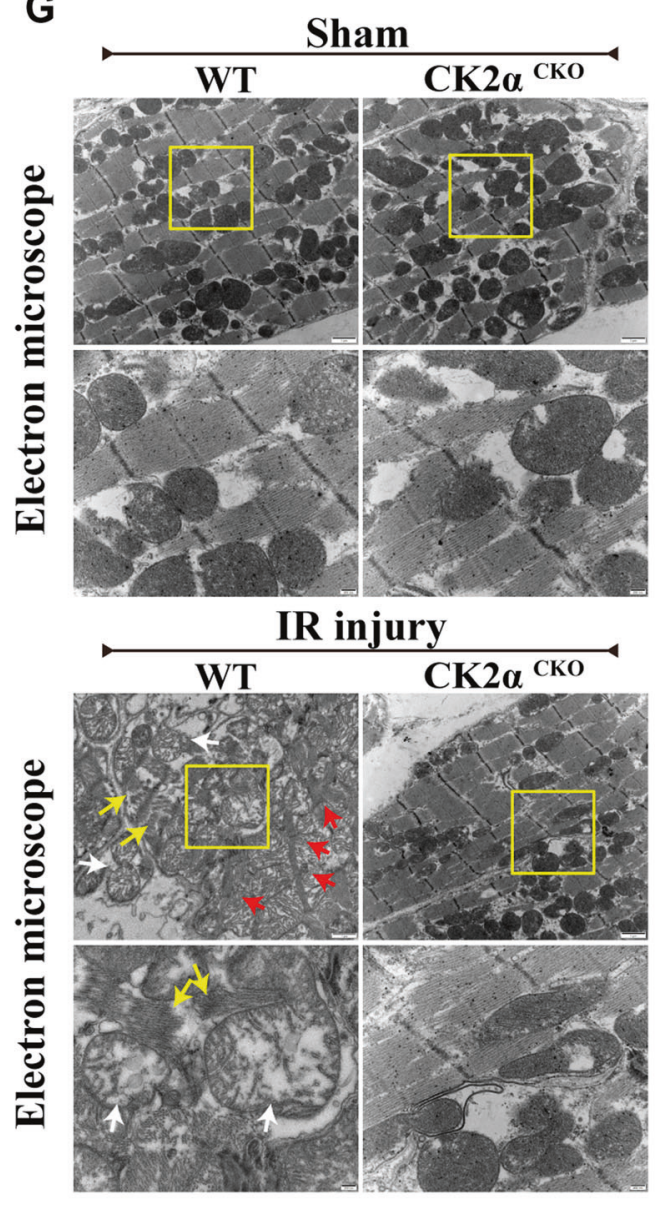

CK2 $\alpha^{C K O}$ mice in the context of IR injury $n=70-80$ cells from 2 mice per group and experiments were repeated three times. The data represent the mean \pm SEM. $* P<0.05$. $+\mathrm{dL} / \mathrm{dt}$ is the maximal velocity of shortening, $-\mathrm{dL} / \mathrm{dt}$ maximal velocity of relengthening, FS fractional shortening, LVEF left ventricular ejection fraction, LVDd left ventricular diastolic dimension, TPS time-to-peak shortening, $\mathrm{TR}_{90}$ timeto-90\% relengthening

Whether reperfusion re-instates the phosphorylated inactivation of FUNDC1 and if so, the site of phosphorylation involved in reperfusion-mediated FUNDC1 inhibition remains unknown.

Casein kinase $2 \alpha(\mathrm{CK} 2 \alpha)$, a constitutive Ser/Thr kinase [21], was originally described as a suppressor of FUNDC1 and is linked to FUNDC1 phosphorylation at Ser13 [22]. eactivity during reperfusion is not completely understood. 

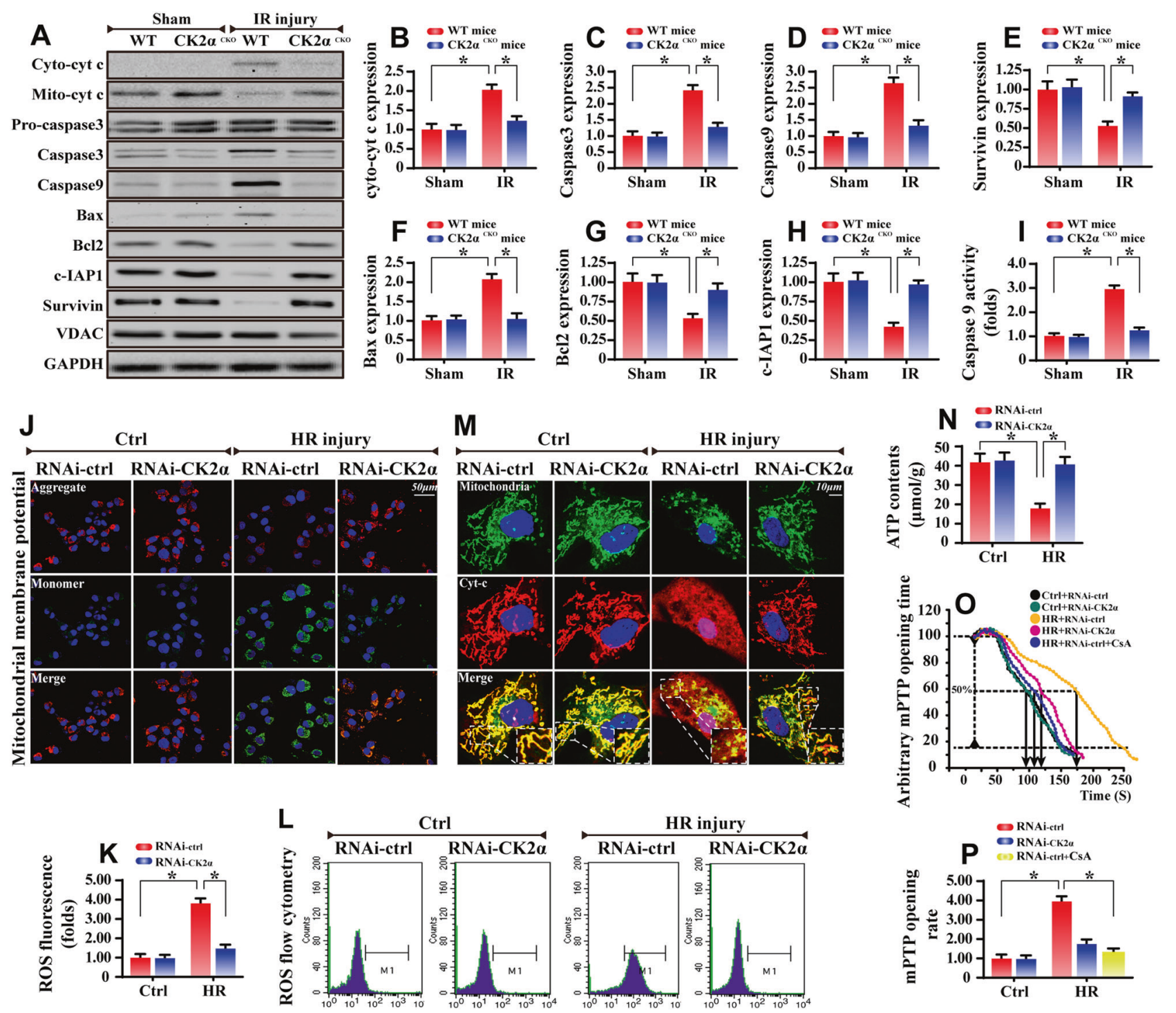

Fig. 3 CK2 $\alpha$ was implicated in cardiac mitochondrial apoptosis. a-h Western blots were used to analyze the proteins alterations related to the mitochondrial apoptosis in vivo. VDAC is the loading control of mitochondrial proteins. $\mathbf{i}$ The caspase 9 activity detection via ELISA assay. j In vitro, JC-1 staining was used to display the collapse of mitochondrial potential. $\mathbf{k}$, l Cellular ROS content was quantified via flow cytometry analysis. m Immunofluorescence of cyt-c.

Mitochondria were marked by Tom20. Nuclei were labeled by DAPI. n ATP production was analyzed via commercial kit. o, p mPTP opening time was determined as the time when the TMRE fluorescence intensity decreased by half between the initial and residual fluorescence intensity. CsA (Cyclosporine A) was used as the negative control group. The data represent the mean \pm SEM. $* P<0.05$

Solid evidence has illustrated that CK2 $\alpha$ is implicated in the development of diabetic cardiomyopathy [23] and heart failure [24]. What remains unclear is if $\mathrm{CK} 2 \alpha$ contributes to the progression of cardiac IR injury and, if so, whether this is governed by $\mathrm{CK} 2 \alpha$-elicited mitochondrial injury and cardiomyocyte damage through inhibition of mitophagy mediated via FUNDC1 phosphorylation at Ser13 during reperfusion. Herein, this study aims to explore the role of CK2 $\alpha$ and FUNDC1-required mitophagy in the development of cardiac IR injury.

\section{Results}

\section{CK2 $a$ is activated by cardiac IR stimulation and promotes the development of IR injury}

To observe the cardiac CK $2 \alpha$ response to IR injury, mice were subjected to $45 \mathrm{~min}$ ischemia followed by $0-24 \mathrm{~h}$ of reperfusion. Our results suggested that $\mathrm{CK} 2 \alpha$ transcription (Supplemental Fig. 1A) and expression (Fig. 1a, b) were progressively increased during the course of reperfusion. 
Notably, when compared to the normoxic and/or ischemic stages, $30 \mathrm{~min}$ of reperfusion significantly upregulated CK $2 \alpha$ expression. Moreover, CK $2 \alpha$ content was highest following $6 \mathrm{~h}$ of reperfusion. After that, prolonged duration of reperfusion failed to further upregulate CK $2 \alpha$ expression (Fig. 1a, b). Similar results were obtained in cardiomyocytes in the setting of hypoxia and reoxygenation in vitro (Supplemental Fig. 1B-C). Therefore, reperfusion for $6 \mathrm{~h}$ was used in the remaining experiments. Subsequently, we asked whether $\mathrm{CK} 2 \alpha$ had a causal role in regulating IR injury and thus cardiac-specific $\mathrm{CK} 2 \alpha$ knockout $\left(\mathrm{CK} 2 \alpha^{C K O}\right)$ mice were employed. Western blots and immunohistochemistry were used to confirm the CK $2 \alpha$ expression. IR upregulated the CK2 $\alpha$ expression in WT mice but not in CK $2 \alpha^{C K O}$ mice (Fig. 1c-e). Furthermore, CK $2 \alpha^{C K O}$ mice demonstrated a significant reduction in infarction area compared with that in the WT group (Fig. 1f, g). TUNEL assay displayed that $\mathrm{CK} 2 \alpha^{C K O}$ mice had fewer TUNELpositive cells than mice in the WT group (Fig. 1h, i).

More solid evidence was obtained in primary cardiomyocytes with $45 \mathrm{~min}$ hypoxia followed by $6 \mathrm{~h}$ of reoxygenation (HR). CK2 $\alpha$ expression was greatly increased after HR (Fig. 1j, k). Subsequently, the loss-of function assay for CK2 $\alpha$ was carried out using siRNA, and knockdown efficiency was confirmed by western blots (Fig. 1j, k). Silencing of CK $2 \alpha$ displayed no effect on cellular viability although it attenuated HR-induced cellular death as evidenced by casapse 3 activity and MTT assay (Fig. 11, m). To exclude the influence of siRNA transfection on HRmediated cellular damage, cardiomyocytes were also isolated from WT and $\mathrm{CK} 2 \alpha^{C K O}$ mice and were subjected to HR injury in vitro. Through TUENL assay (Supplemental Fig. 1D-E), HR was found to obviously increase TUNELpositive cardiomyocytes isolated from WT mice; the effect of which was abolished in cells obtained from CK $2 \alpha^{C K O}$ mice. These data indicate that upregulated CK2 $\alpha$ likely participated in the IR injury via mediating cellular death.

\section{CK2a deficiency maintains cardiac function}

Outside of cellular death, we further evaluated the changes of cardiac function. Compared with WT mice, the CK $2 \alpha{ }^{C K O}$ mice displayed less amounts of LDH, Troponin T and CKMB (Fig. 2a-c). Meanwhile, the IR-suppressed cardiac function including left ventricular ejection fraction (LVEF) and left ventricular fractional shorting (LVFS) was restored to $\sim 92$ and $\sim 95 \%$, respectively, of the baseline levels, in $\mathrm{CK} 2 \alpha^{C K O}$ mice (Fig. 2d-f). Moreover, left ventricular diastolic dimension (LVDd) was also significantly decreased in CK $2 \alpha^{C K O}$ mice after IR injury when compared to that in IRchallenged WT mice (Fig. 2d-f).

To discern if functional improvement is originated from the structural protection, electron microscope (EM) was used to assess the architecture of myocardium (Fig. 2g). Our results indicate that IR injury triggered more mitochondrial damage (white arrows), myocyte dissolution (yellow arrows), muscular fiber twisting and $\mathrm{Z}$ line disappearance (red arrows); and these changes mostly disappeared in $\mathrm{CK} 2 \alpha^{C K O}$ mice. Subsequently, cardiomyocyte properties were detected through acute isolation of cardiomyocytes from WT and CK2 $\alpha^{C K O}$ mice after IR injury based on our previous study [25]. Neither IR injury nor $\mathrm{CK} 2 \alpha$ deletion overtly affected the resting cell length in cardiomyocytes (Fig. 2h). However, cardiomyocytes from WT mice exposed to IR injury displayed significantly depressed PS and $\pm \mathrm{dL} / \mathrm{dt}$ as well as prolonged TPS and TR To $_{9}$ (Fig. $2 \mathrm{i}-\mathrm{m}$ ). In contrast, $\mathrm{CK} 2 \alpha$ deficiency did not affect these mechanical parameters tested; it apparently reduced or abrogated IR-induced mechanical changes. To exclude the influence of fresh isolation on cellular function, spontaneous contraction was monitored in primary cardiomyocytes isolated from WT mice with or without $\mathrm{CK} 2 \alpha$ siRNA silencing following $\mathrm{HR}$ treatment. Videos (Supplemental videos AD) of cardiomyocytes beating display that loss of CK $2 \alpha$ sustained the rhythm and amplitude cardiomyocyte contraction after HR injury. These data indicate that CK $2 \alpha$ likely promoted cardiac dysfunction in IR injury.

\section{CK2a deficiency affords cardiomyocyte protection against mitochondria-related cell death}

Mitochondrial death in cardiomyocytes is deemed the primary factor for the development of IR injury [26]. As shown in Fig. 3a-i, IR injury upregulates the expression of mitochondrial pro-apoptotic proteins and promotes caspase 9 activity, the effects of which were abrogated by CK2 $\alpha$ deletion. Similar results were also obtained in primary cardiomyocytes with CK2 $\alpha$ siRNA silencing in the setting of HR injury (Supplemental Fig. 2A-H), or in cardiomyocytes isolated from WT and CK2 $\alpha^{C K O}$ mice subject to $\mathrm{HR}$ insult (Supplemental Fig. 2I).

Mitochondrial apoptosis is characterized by $\Delta \Psi \mathrm{m}$ dissipation, cyt-c leakage, mPTP opening, ROS overproduction and ATP undersupply [27]. In vitro, HR treatment reduced the $\Delta \Psi \mathrm{m}$ (Fig. 3j), which was accompanied with more ROS production via flow cytometry analysis (Fig. 3k, 1). HR attack also caused more cyt-c leakage from the mitochondria into the cytoplasm, even into the nucleus, as revealed by immunofluorescence (Fig. $3 \mathrm{~m}$ ). In addition, HR suppressed ATP production (Fig. 3n) and increased the mPTP opening (Fig. 3o, p) in vitro. In contrast, with the loss of $\mathrm{CK} 2 \alpha, \mathrm{HR}$-induced changes in $\Delta \Psi \mathrm{m}$ reduction, ROS overproduction, cyt-c leakage, mPTP opening and ATP shortage were dramatically recused. Mitochondrial energy is vital for cellular survival in response to reperfusion injury. HR reduced the state $3 / 4$ 
A

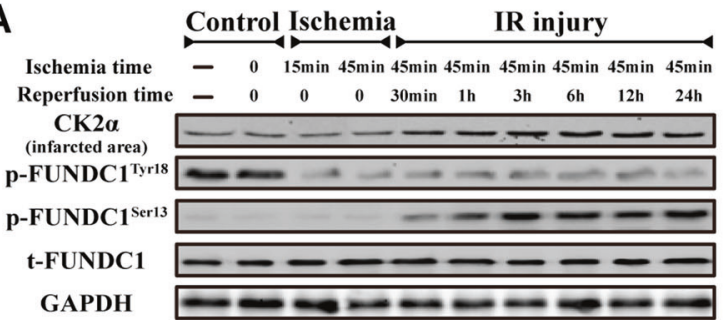

C
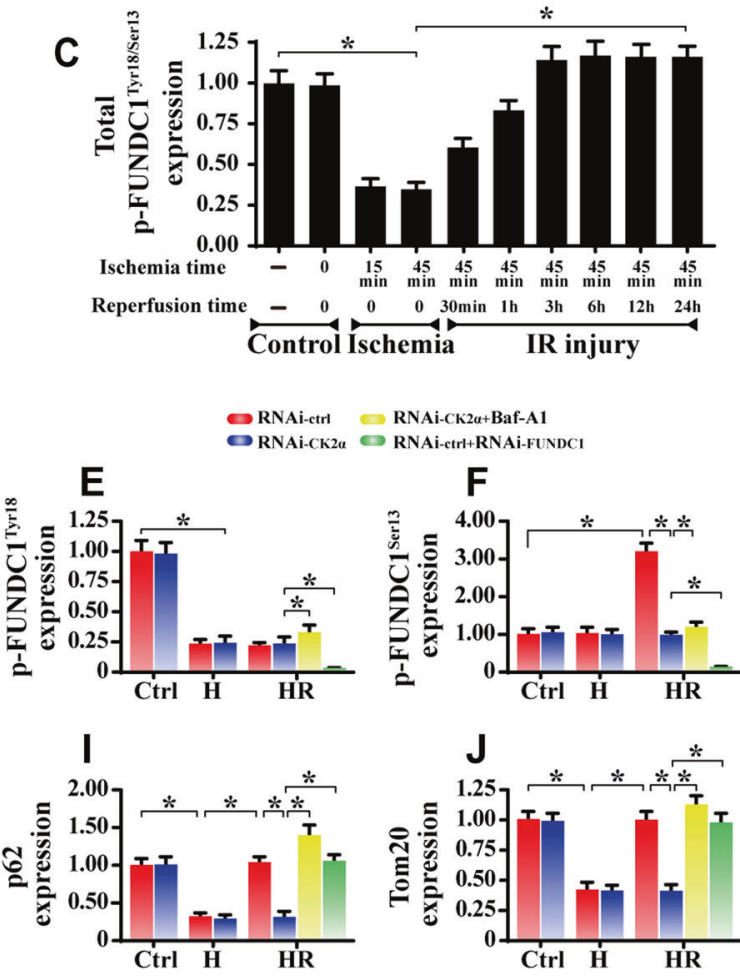
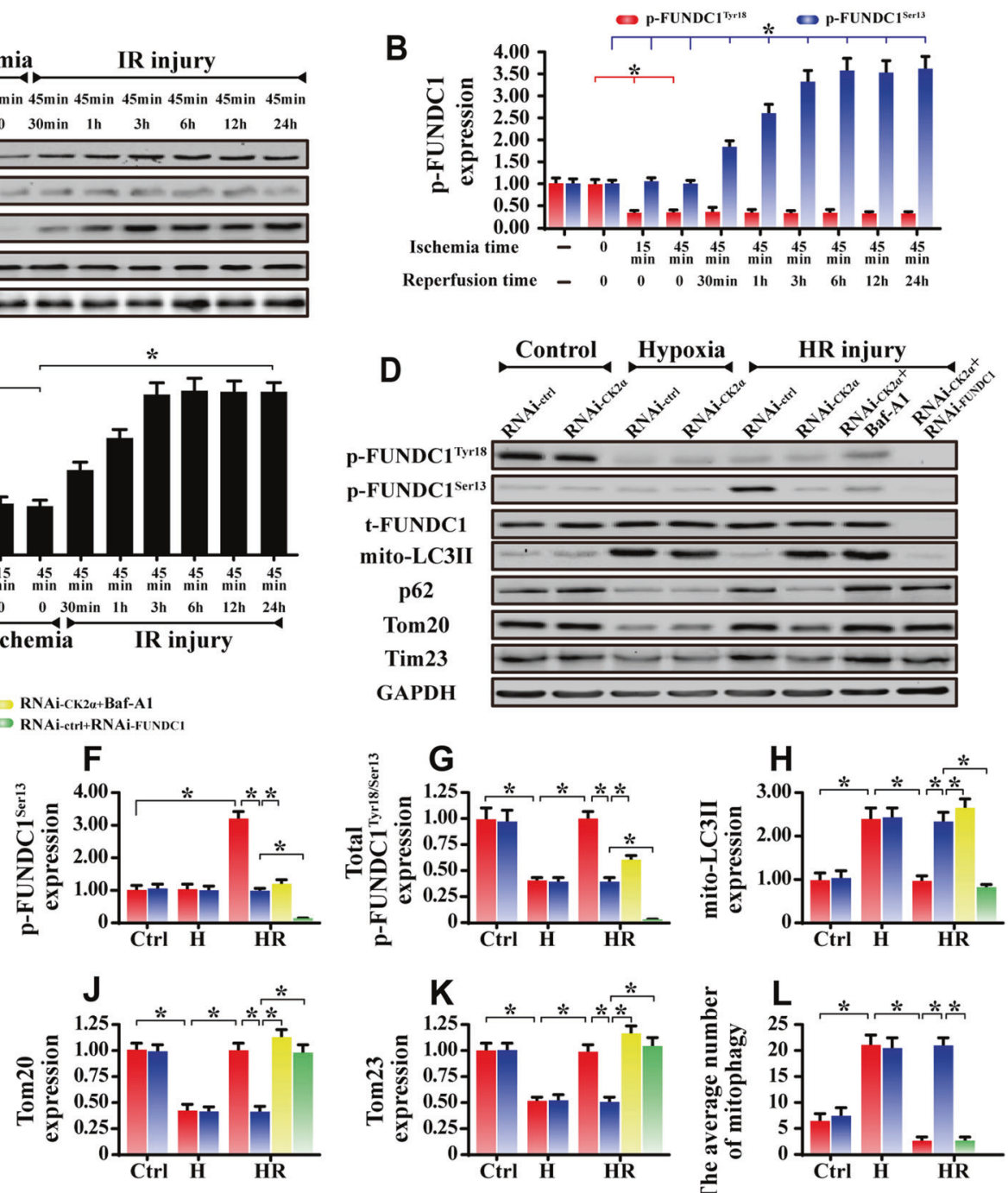
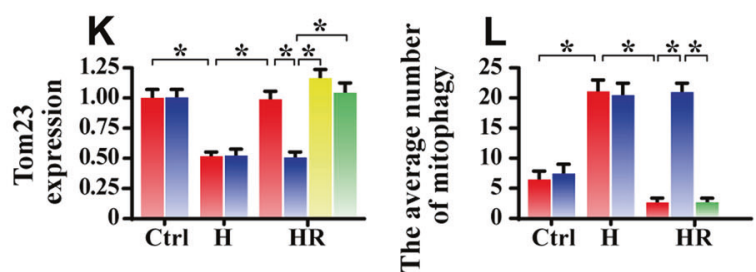

M
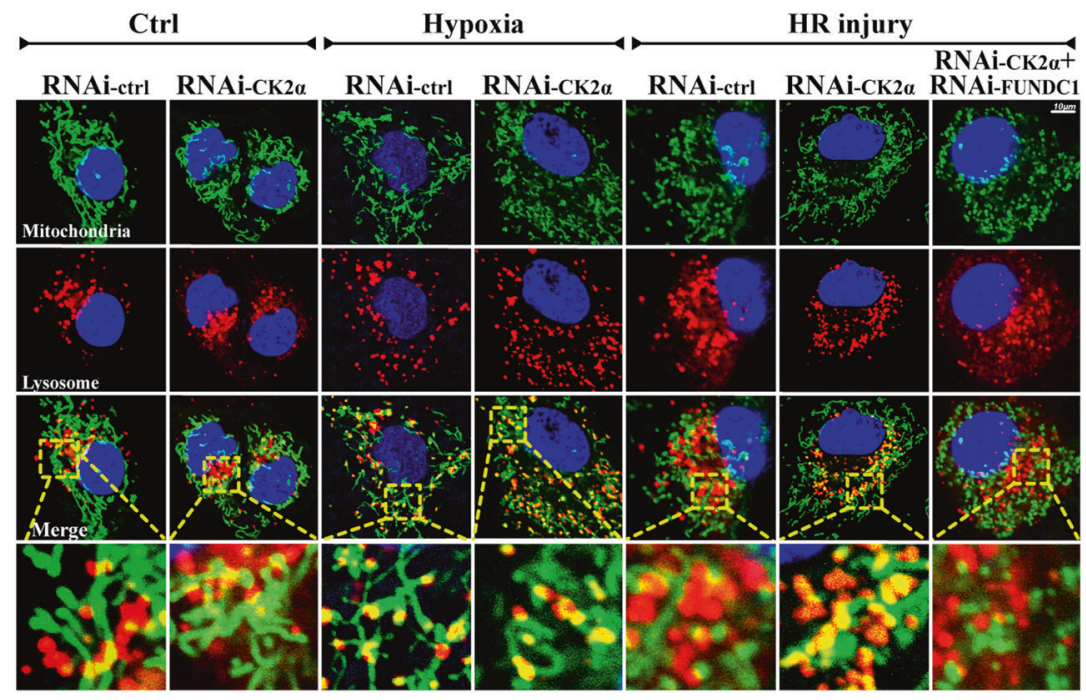

respiratory rates, ADP phosphorylation (respiratory control ratio), efficiency of ATP synthesis (ADP/O) and ADP phosphorylation lag phase (time elapsed in the depolarization/repolarization cycle during ADP phosphorylation) (Supplemental Fig. 2J-N). However, loss of CK2 $\alpha$ reversed the mitochondrial respiratory function. These data 
Fig. 4 CK2 $\alpha$ influenced FUNDC1-required mitophagy. a-c Proteins were isolated from heart tissues under normoxia (control group), ischemia and reperfusion conditions. The FUNDC1 phosphorylation was detected. d-k Proteins were isolated from cardiomyocytes with CK2 $\alpha$ silenced in the presence of normoxia (control group), hypoxia and HR. FUNDC1 phosphorylation and mitophagy markers were analyzed via western blots. siRNA against FUNDC1 was used to establish the loss of function assay examining the role of FUNDC1 in CK2 $\alpha$-deleted cells. Bafilomycin-A1 (Baf-A1), the inhibitor of autophagolysosome, was used to evaluate the autophagy flux. $\mathbf{l}, \mathbf{m}$ To directly observe changes in mitophagy, the co-immunofluorescence of mitochondria and lysosomes was used. Organ immunofluorescence was the hallmark of the interaction of mitochondria and lysosomes and was indicative of mitophagy. The data represent the mean \pm SEM. $* P$ $<0.05$

substantiated that mitochondria are likely the target of CK2 $\alpha$ under IR injury.

\section{FUNDC1-required mitophagy is upregulated in response to CK2a deletion}

FUNDC1-mediated mitophagy is beneficial for cardiac IR injury. However, phosphorylated FUNDC1 exerts steric hindrance for LC3II binding [18], thus strongly inhibiting mitophagy. As shown in Fig. 4a, b, at normoxia condition (control group), abundant p-FUNDC1 ${ }^{\text {Tyr18 }}$ were predominately found in cardiomyocytes whereas subtle pFUNDC $1{ }^{\text {Ser13 }}$ expression was observed, indicating that baseline FUNDC1 activity was primarily governed by $\mathrm{p}$ FUNDC $1^{\text {Tyr18 }}$. In response to the ischemia, the level of $\mathrm{p}$ FUNDC $1^{\text {Tyr18 }}$ was dramatically downregulated whereas $\mathrm{p}$ FUNDC $1{ }^{\text {Ser13 }}$ remained relatively unchanged, indicative of FUNDC1 activation by ischemia via p-FUNDC1 $1^{\text {Tyr18 }}$ downregulation. Furthermore, upon reperfusion exposure, ischemia-inhibited $\mathrm{p}$-FUNDC1 $1^{\mathrm{Ty} 18}$ remained relatively unchanged whereas $\mathrm{p}$-FUNDC1 $1^{\mathrm{Ser} 13}$ was gradually upregulated, indicative of FUNDC1 inactivation by reperfusion via $\mathrm{p}-\mathrm{FUNDC1}{ }^{\text {Ser13 }}$ upregulation. Through analysis of the levels of phosphorylated FUNDC1 $1^{\text {Tyr18/Ser13 }}$ (Fig. 4a, c), we demonstrated that the inactivated forms of FUNDC1 (total levels of $\mathrm{p}-\mathrm{FUNDC} 1^{\mathrm{Ty} 18 / \mathrm{Ser} 13}$ ), were firstly downregulated during ischemia but then were progressively upregulated in reperfusion. Given that no alterations were noted for $\mathrm{p}$ FUNDC1 $1^{\text {Tyr18 }}$ at both ischemia and reperfusion stages, the increase in total levels of p-FUNDC $1^{\text {Tyr18/Ser13 }}$ in reperfusion phase is primarily attributable to enhanced pFUNDC $1{ }^{\text {Ser13 }}$. Similar results were also found in cardiomyocytes in vitro (Supplemental Fig. 3A-D).

The synchronous changes of CK2 $\alpha$ and p-FUNDC1 $1^{\text {Ser13 }}$ in reperfusion, in conjunction with the fact that $\mathrm{p}$ FUNDC $1^{\text {Ser13 }}$ is the substrate of $\mathrm{CK} 2 \alpha$, implies that reperfusion-upregulated $\mathrm{CK} 2 \alpha$ may interrupt mitophagy through enhanced p-FUNDC1 ${ }^{\text {Ser13 }}$. Through loss-of-function assay of CK2 $\alpha$, we demonstrate that p-FUNDC $1^{\text {Tyr18 }}$ level is only controlled by hypoxia. CK2 $\alpha$ silence had no effects on its content regardless of normoxia, hypoxia and reoxygenation (Fig. 4d-g). In comparison, little p-FUNDC1 ${ }^{\text {Ser13 }}$ level was identified under normoxia and hypoxia conditions. Its expression was mainly increased by reoxygenation; this effect was reversed by CK2 $\alpha$ knockdown (Fig. $4 d-g$ ). These data illustrate that baseline CK2 $\alpha$ failed to induce FUNDC1 phosphorylation (inactivation) whereas reperfusionupregulated CK2 $\alpha$ exclusively promoted FUNDC1 phosphorylation at Ser13.

Furthermore, mitophagy activity is also elevated under hypoxia as evidenced by higher mito-LC3II, less p62 and lower Tim23 (mitochondrial inner membrane marker) and Tom20 (outer membrane marker) (Fig. 4d, h-k). In contrast, reoxygenation unfortunately repressed mitophagy, the effect of which was nullified by CK2 $\alpha$ deletion (Fig. $4 \mathrm{~d}$, h-k). Through analysis of the mitophagic flux using bafilomycinA1 (Baf-A1), we re-confirmed that $\mathrm{CK} 2 \alpha$ deletion was able to augment mitophagy activity (Fig. 4d-k). Interestingly, under loss of FUNDC1, the beneficial role of CK2 $\alpha$ deficiency in mitophagy activation disappeared (Fig. $4 \mathrm{~d}-\mathrm{k}$ ). Similar results were also obtained in WT and CK $2 \alpha^{C K O}$ mice (Supplemental Fig. 4A-F).

To directly assess mitophagy activity, immunofluorescence assay of mitochondria and lysosome was used (Fig. 4l, m). Most fragmented mitochondria cannot be engulfed by lysosomes following HR treatment, as demonstrated by sporadic co-localization of mitochondria and lysosomes, indicating mitophagy inhibition. These changes were reversed by CK2 $\alpha$ deletion, which contributed to the fusion of mitochondria and lysosomes. Furthermore, once FUNDC1 expression was lost, the overlap of lysosome and mitochondria was re-inhibited in CK2 $\alpha$ deleted cells. Similar results were also observed in cardiomyocytes isolated from WT and CK2 $\alpha^{C K O}$ mice (Supplemental Fig. 4G-H).

\section{Genetic inhibition of FUNDC1 abolishes the protection observed in $\mathrm{CK}_{2} a^{\mathrm{CKO}}$ mice}

To further confirm whether FUNDC1 activity is required for the protective effect of CK2 $\alpha$ depletion in vivo, CK $2 \alpha-$ FUNDC1 double-deficient mice were generated. Expected reduction in CK2 $\alpha$ and FUNDC1 proteins were confirmed using western blot (Fig. 5a-c), and then these mice were subjected to IR injury. Consistent with our findings above, the $\mathrm{CK} 2 \alpha^{C K O}$ mice had reduced infarcted area (Fig. $5 \mathrm{~d}$, e), improved cardiac function (Fig. 5f, g, j-1) and less TUNEL ${ }^{+}$ cells (Fig. 5h, i) when compared to the WT mice under IR injury. Interestingly, the CK2 $\alpha$-FUNDC1 double-deficient mice displayed increased infarcted zone, impaired cardiac function and more abundant cell death compared with CK $2 \alpha^{C K O}$ mice (Fig. 5d-1). As depicted in our EM 

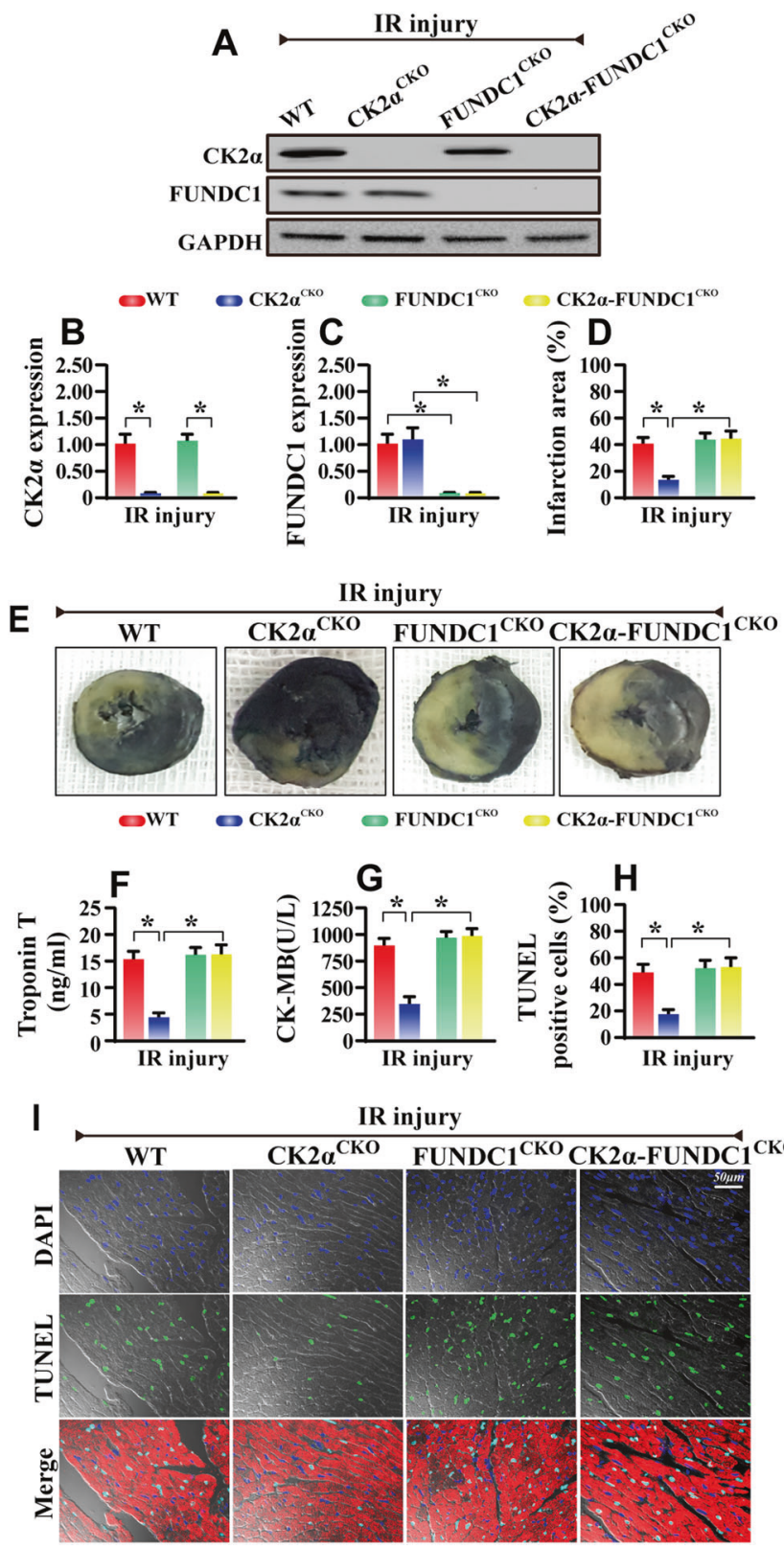

Fig. 5 Deletion of cardiac FUNDC1 abrogated the cardio-protection observed in CK2 $\alpha^{C K O}$ mice under IR. a-c Western blots was used to confirm the proteins changes in $\mathrm{CK} 2 \alpha^{C K O}$ mice, FUNDC1 ${ }^{C K O}$ mice and CK $2 \alpha$-FUNDC1 ${ }^{C K O}$ mice. d, e The infarction area was measured after 45-min ischemia and 6-hour reperfusion. $\mathbf{f}, \mathbf{g}$ The cardiac damage markers were detected via ELISA assay. h, i The cellular apoptosis was evaluated via TUNEL assay. The red fluorescence is the staining

ultrastructural evaluation, genetic ablation of FUNDC1 reinduced mitochondrial vacuolization and myocyte dissolution when compared to the $\mathrm{CK} 2 \alpha^{C K O}$ mice (Supplemental Fig. 5A).

Similarly, in vitro, we silenced FUNDC1 in CK2 $\alpha$ deleted cardiomyocytes, and the results indicate that the loss of FUNDC1 in CK2 $\alpha$-deleted cardiomyocytes caused more cell death via mitochondrial damage, as evidenced by more
WT
CK2a

K

$\mathbf{L}$
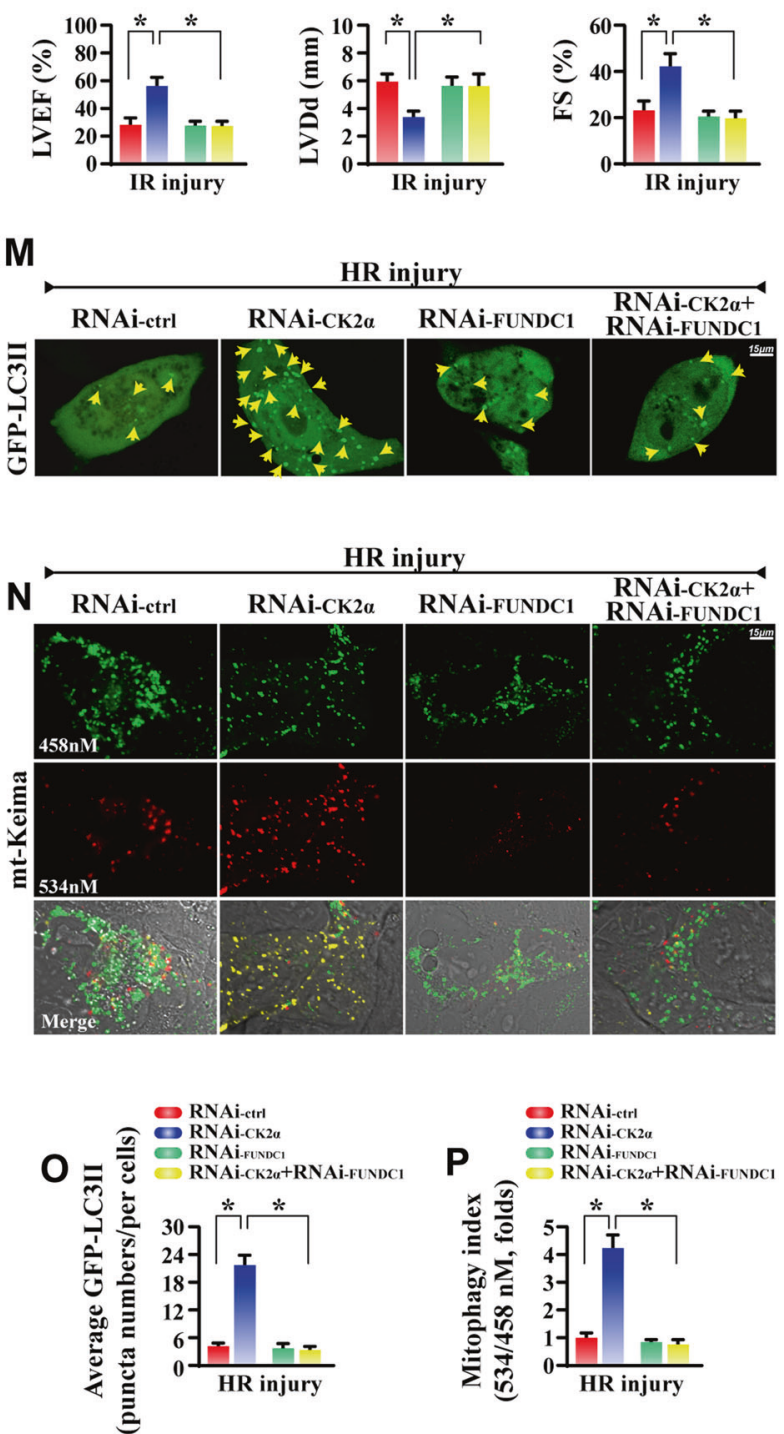

of Troponin T. $\mathbf{j}-\mathbf{l}$ The cardiac function was detected via echocardiography. m, o GFP-LC3 transfection was used to detect mitophagy activity. The LC3II puncta were counted. n, p The mt-Keima assay was used to detect the acid mitochondria which is the result of fusion of mitochondria and lysosome. The orange mitochondria were counted. The data represent the mean \pm SEM. $* P<0.05$

TUNEL $^{+}$cells (Supplemental Fig. 5B-C), increased caspase3 activity (Supplemental Fig. 5D) and more mitochondrial pro-apoptotic proteins (Supplemental Fig. 5E-I).

As for mitophagy, through the GFP-LC3 transfection, we demonstrate that less LC3II accumulates in HR-treated cells, and this change were recused by $\mathrm{CK} 2 \alpha$ deletion (Fig. $5 \mathrm{~m}, \mathrm{o}$ ). In contrast, with the knockdown of FUNDC1 in CK2 $\alpha$-deficiency cells, the number of punctate LC3II is 

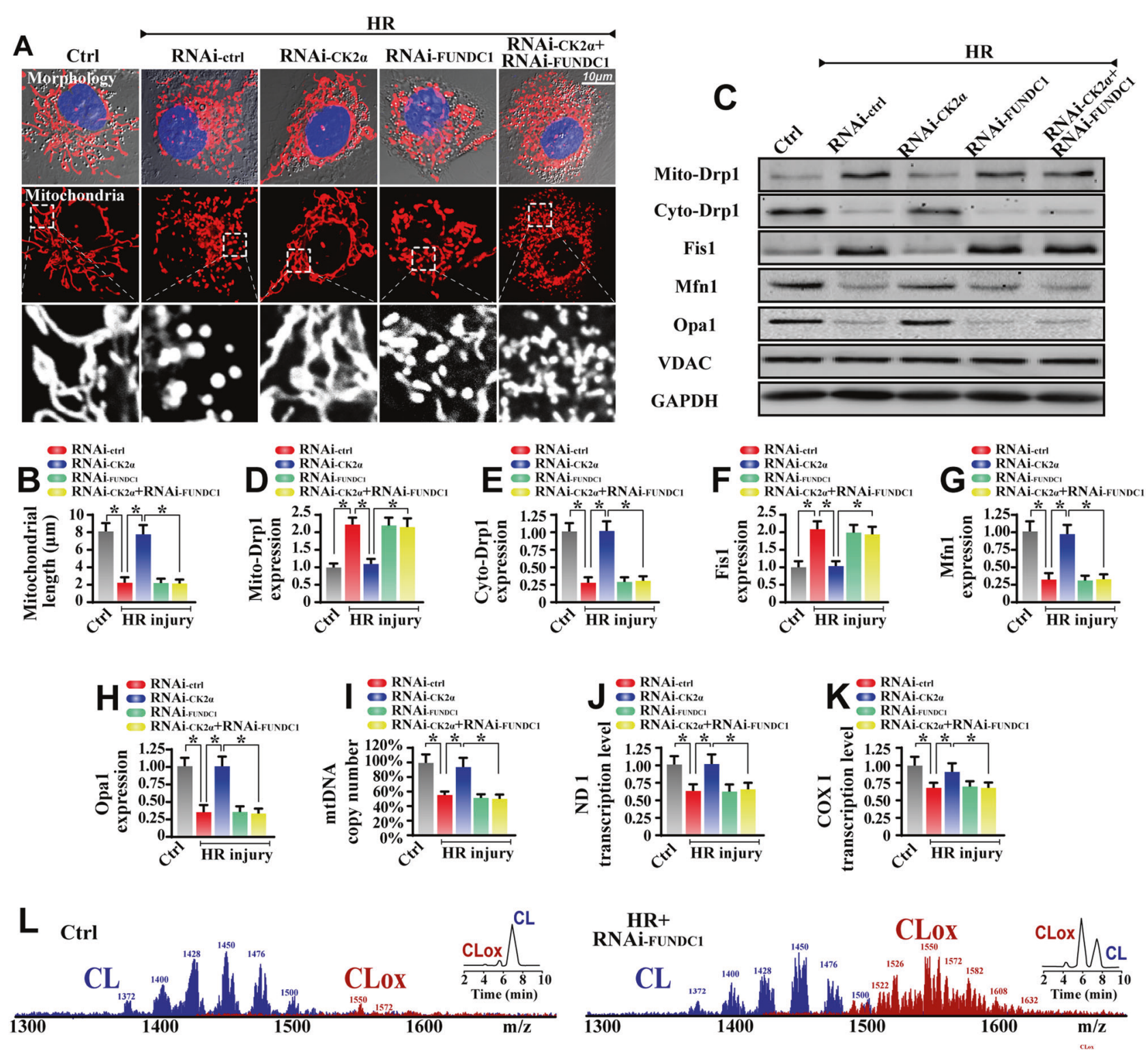

HR+
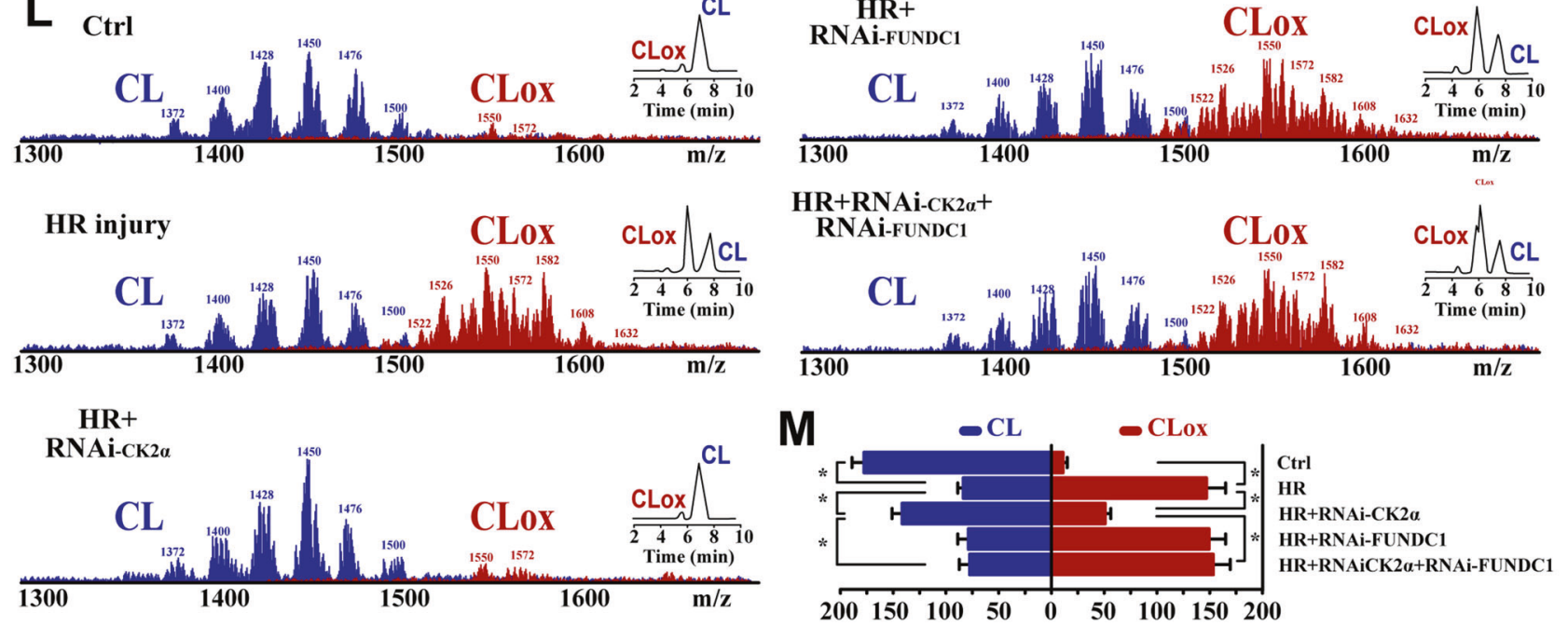

Fig. 6 FUNDC1-required mitophagy protected the mitochondrial homeostasis under IR injury. a Cardiomyocytes were labeled with Tom 20 to determine mitochondrial fragmentation. b To assess changes in mitochondrial morphology quantitatively, the length of mitochondria was measured. $\mathbf{c}-\mathbf{h}$ Proteins related to mitochondrial fission and fusion were evaluated via western blots. i mtDNA copy number was assessed by complex IV segment. $\mathbf{j}$, $\mathbf{k}$ The transcript level of mtDNA

was reflected by two different components: NADH dehydrogenase subunit 1 (ND1) and cytochrome c oxidase subunit I (COX I). I, m Assessment of molecular species of $\mathrm{CL}$ and its oxidation products. The left panel indicates the non-oxidized (blue) and the appearance of numerous oxidized (red) CL species after HR attack. Right insets: 2dimensional chromatographic separation of non-oxidized and oxidized CL (CLox). The data represent the mean \pm SEM. $* P<0.05$ 
reduced again (Fig. $5 \mathrm{~m}, \mathrm{o}$ ). To provide more evidence for mitophagy activity, mt-Keima assay was used. More acid mitochondria were found in CK2 $\alpha$-deleted cells compared to the HR group. However, loss of FUNDC1 abated the number of acid mitochondria despite deletion of CK2 $\alpha$ (Fig. 5n, p). These data demonstrate that FUNDC1 was necessary for the beneficial action of $\mathrm{CK} 2 \alpha$ deletion via mitophagy.

\section{FUNDC1-required mitophagy protects mitochondrial structure and function}

To explore the possible protective role of FUNDC1 in IR injury, mitochondrial fragmentation was monitored based on the above observation that more round mitochondrial debris appeared in HR-treated cells. As shown in Fig. 6a, b, after treatment with HR, mitochondria divided into several fragments and that this configuration change was reversed by CK $2 \alpha$ deletion. Furthermore, loss of FUNDC1 would reinduce mitochondrial debris in CK2 $\alpha$-deleted cells. Furthermore, the proteins related to mitochondrial fission including dynamin-related protein 1 (Drp1) and mitochondrial fission protein 1 (Fis1) were increased in HR treatment but reduced in CK2 $\alpha$-deleted cells (Fig. 6c-h). However, loss of FUNDC1 re-instated the accumulation of mito-Drp1 and Fis 1 despite deletion of CK2 $\alpha$. On the other hand, the mitochondrial fusion proteins such as mitofusin 1 (Mfn1) and optic atrophy 1 (Opa1) were reduced in HR treatment but reversed to normal levels in CK2 $\alpha$-deleted cells in a FUNDC1-dependent manner (Fig. 6c-h). Similar results were also found in vivo experiments (Supplemental Fig. 6). These data indicate that loss of CK2 $\alpha$ inhibits fission and promotes fusion via FUNDC1-required mitophagy which powerfully cleared mitochondrial fragmentations.

The key feature of mitochondrial homeostasis is mitochondrial genome stability. However, the mtDNA copy number (Fig. 6i) and mtDNA transcripts (Fig. 6j-k) were downregulated in HR-treated cardiomyocytes, and these changes were reversed by CK2 $\alpha$ deletion. However, upon knockdown of FUNDC1 in CK2 $\alpha$-deleted cells, the mtDNA copy and transcription were reduced again. The electron transport chain complexes' (ETC) activity is mainly handled by mtDNA. However, HR reduced the activity of ETC (Supplemental Fig. 7A-C), which was reversed via CK2 $\alpha$ deletion, whereas inhibition of FUNDC1 canceled the protective action of $\mathrm{CK} 2 \alpha$ deletion.

As for mitochondrial damage, we focused on cardiolipin (CL) oxidation which occurs at the early stage of mitochondrial apoptosis via liberation of cyt-c into cytoplasm. Using 2-dimensional high-performance thin-layer chromatography, we performed a global lipidomic analysis of CL (Fig. 61, m), which demonstrated that of $\approx 185$ individual molecular species of CL in normal cardiomyocytes, only $\approx 10$ were oxygenated. Notably, HR induced the oxidation of the majority of the polyunsaturated molecular species of $\mathrm{CL}$; the number of non-oxidized CL species decreased to $\approx 94$, whereas the number of oxygenated species increased to $\approx 154$ (Fig. $61, \mathrm{~m}$ ). Loss of CK2 $\alpha$ reversed the ratio of nonoxidized CL, and these effects were nullified by FUNDC1 knockdown. Similar results were obtained via mitochondrial potential staining (Supplemental Fig. 7D-E), ROS oxidative stress detection (Supplemental Fig. 7F-G), mPTP opening assay (Supplemental Fig. 7H), and mitochondrial apoptotic proteins measurement (Supplemental Fig. 7I-M). These data indicate that loss of FUNDC1-related mitophagy aggravates mitochondrial damage.

Considering that the mitochondrial mass was reduced whereas the mtDNA copy/transcription was increased, which was accompanied with the decline in oxidized CL, we guessed whether FUNDC1-required mitophagy promoted the mitochondrial biogenesis. As shown in Supplemental Fig. 7N-P, peroxisome proliferator-activated receptor gamma coactivator 1-alpha (PGC1a), nuclear respiratory factor 1 (NRF1), and mitochondrial transcription factor A (TFAM) transcriptions were reduced in HR-treated cells but increased in CK2 $\alpha$-deleted cells. Interestingly, FUNDC1 deficiency re-evoked a deep decline in PGC1a, NRF1, and TFAM transcriptions, suggesting that FUNDC1related mitophagy promoted the mitochondrial turnover via upregulation of mitochondrial biogenesis.

\section{Discussion}

In this study, we uncover that (1) CK2 $\alpha$ is progressively increased in the reperfused heart, which is instrumental for scar expansion and cardiac dysfunction in response to IR injury; (2) functional assays illustrate that higher CK2 $\alpha$ expression renders cardiomyocytes to mitochondrial apoptosis via post-transcriptional inactivation of FUNDC1, leading to the inhibition of protective mitophagy; (3) defective mitophagy fails to remove the damaged mitochondria induced by IR injury, resulting in mitochondrial genome collapse, ETC inactivity, cardiolipin oxidation and mitochondrial apoptosis; (4) loss of cardiac CK2 $\alpha$ sustains heart function and structure under IR injury, however, (5) genetic ablation of FUNDC1 abolishes the protection observed in CK2 $\alpha$ deletion in vivo and in vivo. Collectively, IR promoted disturbance in mitochondrial integrity, thus demanding well-orchestrated mitophagy to remove damaged mitochondria. Unfortunately, FUNDC1-required mitophagy was suppressed via reperfusion by way of CK2 $\alpha$, leading to the progression of IR injury. As far as we know, this is the first study to identify CK2 $\alpha$-FUNDC1 mitophagy pathways as the pathogenesis of cardiac IR injury via governing mitochondrial homeostasis. 
Mitochondrial damage is conclusively involved in cardiac IR injury via multiple mechanisms [26]. The key finding in the present study is that CK2 $\alpha$ is a novel molecular basis for disturbed mitochondrial homeostasis in IR injury. Protein kinase CK2 $\alpha$ participates in heart and neural tube development, maintains cell viability, and regulates cell cycle stages $[28,29]$. Many studies have demonstrated that $\mathrm{CK} 2 \alpha$ regulates the mitochondrial oxidative [30] and induces p53-related mitochondrial apoptosis [31]. Although previous studies have confirmed the harmful effects of CK2 $\alpha$ on chronic cardiac injury including diabetic cardiomyopathy [23] and heart failure [24], its detailed molecular machinery related to mitochondrial etiology in cardiac IR injury remains largely unexplored. In the present study, we demonstrate that $\mathrm{CK} 2 \alpha$ is activated by IR injury contributing to FUNDC1 inactivation and mitophagy arrest. This finding introduces a role for CK2 $\alpha$-mediated mitophagy in acute cardiac damage for the first time, which should help to fill the gap in the current knowledge regarding the molecular links between $\mathrm{CK} 2 \alpha$, mitophagy, mitochondrial dysfunction and cardiac IR injury.

The harmful insults of CK2 $\alpha$ on mitochondria depends on FUNDC1-related mitophagy activity. First, reperfusion injury definitely induced mitochondrial damage via promoting mitochondrial fission [32], impairing the mtDNA genome stability [33], reducing ETC activity, evoking mitochondrial oxidative stress [34], and more severely, initiating mitochondrial apoptosis $[35,36]$. Despite damage to mitochondria, cells could utilize well-orchestrated mitophagy to timely remove the bad mitochondria and largely sustain the mitochondrial respiratory function [37]. Although FUNDC1required mitophagy has been reported to be activated by ischemia/hypoxia [16, 22, 38], its activity was unfortunately downregulated in reperfusion through poorly defined mechanisms [3, 39]. Our findings propose that FUNDC1required mitophagy was activated by ischemia via $p$ FUNDC $1^{\text {Tyr18 }}$ downregulation but inhibited by reperfusion through upregulated CK2 $\alpha$ and its substrate p-FUNDC1 ${ }^{\text {Ser13 }}$, redefining the paradigm of FUNDC1/mitophagy activation and inactivation during ischemia and reperfusion, respectively. Thereby, this information clearly explains the molecular mechanism responsible for the defective FUNDC1required mitophagy in the context of reperfusion injury.

The process of mitophagy activation relies on a growing cadre of "mitophagy adaptors", and three regulators have been reported according to our previous findings: FUNDC1 [3, 7], Bnip3 [6, 8, 9] and Parkin [14]. More importantly, different receptors may have various influences on cell fate, ranging from cellular protection to cellular self-destruction. In answer to cardiac IR injury, excessive Parkin- and Bnip3involved mitophagy exacerbated cardiomyocytes death $[9$, 14] whereas FUNDC1-related mitophagy provides beneficial effects on cell survival [3, 7]. A recent study [40] has demonstrated that CK2 $\beta$ deletion was associated with mitochondrial dysfunction and activated Parkin-mediated mitophagy due to the impairment of PINK1 importing into mitochondria and blocking autophagic flux. Their finding is similar to our previous study that excessive Parkin-induced mitophagy augmented mitochondrial injury. Accordingly, the discrepancy existing with regard to the detailed role of mitophagy in mitochondrial homeostasis is principally attributed to the various mitophagy adaptors.

At molecular level, FUNDC1 signals mitophagy to sweep the mitochondrial debris, sustain mitochondrial respiratory and block mitochondrial apoptosis. More importantly, although FUNDC1-required mitophagy promotes the clearance of mitochondrial mass, the mtDNA copy/transcription is actually increased, which was accompanied with the elevated mitochondrial biogenesis, leading to the mitochondrial turnover or renewing. These results correlate well with previous conclusion that mitophagy is required for mitochondrial biogenesis via accelerating mitochondria turnover [41, 42]. However, the exact mechanism by which mitophagy promotes the mitochondrial biogenesis remains unclear.

In conclusion, we answer the question of how CK2 $\alpha$ aggravates the development of IR injury: CK2 $\alpha$ cuts off FUNDC1-reqiured mitophagy and impairs the mitochondrial protective system, eventually amplifying the cardiomyocytes death signals.

\section{Methods}

\section{Animal models of cardiac IR Injury}

All animal procedures were performed in accordance with the Guide for the Care and Use of Laboratory Animals, which was published by the US National Institutes of Health (NIH Publication No. 85-23, revised 1996) and were approved by the University of Wyoming Institutional Animal Use and Care Committee (Laramie, WY, USA). We first generated $\mathrm{CK} 2 \alpha^{f l / l}$ mice as previously reported [43]. The detailed information is described in the supplemental methods. The generation of FUNDC1 $1^{f / f l}$ mice was as our previous study described. The cardiac-specific FUNDC1 knockout (FUNDC1 ${ }^{C K O}$ ) and $\mathrm{CK} 2 \alpha$ knockout $\left(\mathrm{CK} 2 \alpha^{C K O}\right)$ mice were generated via CK2 $2 \alpha^{A / f l}$ or FUNDC $1^{f / f l}$ mice with $\alpha$-MHC (alpha myosin heavy chain)-Cre transgenic mice. Subsequently, FUNDC1 ${ }^{f / f l}$ were bred to $\mathrm{CK} 2 \alpha^{C K O}$ mice to generate cardiac-specific CK2 $\alpha$-FUNDC1 double knockout $\left(\mathrm{CK} 2 \alpha-\mathrm{FUNDC} 1^{C K O}, \mathrm{CK} 2 \alpha^{f / f} ; \quad \mathrm{FUNDC}^{f / f /} ; \mathrm{Cre}^{\alpha-M H C}\right)$ mice. To avoid potential variation related to gender, all experiments were performed in male mice. These mice ( 8 weeks old) were used to induce an IR injury model (45 min ischemia and $0-24 \mathrm{~h}$ reperfusion) according to our previous studies [2]. Upon the completion of the 
reperfusion, the hearts were stained with $2 \%$ Evans Blue and 1\% 2,3,5-triphenyltetrazolium chloride. The infarct size was expressed as a percentage of the risk zone ( $n=6 /$ group in one experiment). The content of lactate dehydrogenase (LDH), Troponin $\mathrm{T}$ and creatine kinase-MB (CK-MB) in the blood was evaluated via ELISA assays as our previous study described [2]. Details on echocardiogram, cell shortening/relengthening assay, electron microscopy and TUNEL assay are described in the supplemental methods.

\section{Cell culture, HR injury induction and siRNA assay}

The HR injury model was mimicked in vitro by $45 \mathrm{~min}$ of hypoxia and $6 \mathrm{~h}$ of reoxygenation. The primary cardiomyocytes were isolated from WT and $\mathrm{CK} 2 \alpha^{C K O}$ mice according to our previous study [44]. To inhibit the mitophagy, siRNA against FUNDC1 was transfected to cardiomyocytes isolated from WT mice. To observe the autophagic flux, Bafilomycin-A1 $(0.5 \mu \mathrm{M}$, Selleck Chemicals) was used $12 \mathrm{~h}$ before treatment. Details on MTT assay, TUNEL staining and caspase $3 / 9$ activities are described in the supplemental methods. The siRNAs specific against the expression of CK2 $\alpha$ and FUNDC1 or control siRNAs were purchased Santa Cruz Biotechnology. The siRNA transfection was based on our previous study [45], and the transfection efficiency was confirmed by western blots.

\section{Mitophagy detection}

Mitophagy was observed via immunofluorescence by costaining of mitochondria and lysosome. The mitophagy flux was measured by detecting GFP-LC3II puncta through transfection of recombinant adenovirus GFP-LC3 under laser scanning confocal microscopy (Nikon A1R, Japan). MtKeima is a ratiometric $\mathrm{pH}$-sensitive fluorescent protein that is targeted into the mitochondrial matrix. A low-ratio mt-Keima derived fluorescence $(543 / 458 \mathrm{~nm})$ reports neutral environment, whereas a high-ratio fluorescence reports acidic $\mathrm{pH}$. Thus, mt-Keima enables differential imaging of mitochondria in the cytoplasm and mitochondria in acidic lysosomes. Mitochondria-targeted mKeima-Red expression plasmid (pMT-mKeima-Red, \#AM-V-251, MBL Medical \& Biological Laboratories, Co., ltd. Woburn, MA) was transfected to cells. Ratio $(543 / 458 \mathrm{~nm})$ of mKeima emission light were calculated as a value of mitophagy. Details on ROS measurement, ATP production, and mitochondrial respiratory function are described in the supplemental methods.

\section{Western blotting and immunofluorescence}

To detect the protein expression, immunoblotting was carried out. Briefly, samples were lysed in RIPA buffer containing 1 mM PMSF. After centrifugation, 30-60 $\mu \mathrm{g}$ protein for each sample was loaded and transferred to PVDF membrane. For immunofluorescence, samples were firstly washed with PBS, and then fixed with $4 \%$ paraformaldehyde for $30 \mathrm{~min}$. Subsequently, samples were incubated with the primary antibody at $4{ }^{\circ} \mathrm{C}$ overnight. Then, samples were stained with fluorescent second antibody for $30 \mathrm{~min}$. DAPI was used for nuclear staining. The pictures were acquired under confocal microscopy. The primary antibodies used in the present study are described in the supplemental methods.

\section{Cardiolipin extraction and high-performance thin- layer chromatography analysis}

The mitochondria were used to obtain cardiolipin by highperformance thin-layer chromatography with an electrospray ionization source and a linear ion trap mass spectrometer (LXQ Thermo-Fisher). To avoid the oxidation of phospholipids during separation, chloroform/methanol (2/1, $\mathrm{v} / \mathrm{v}$ ) containing $0.05 \%$ BHT as antioxidant was added. The cardiolipin and its oxidized molecular species were extracted, separated and analyzed based on our report [2].

\section{The mtDNA copy/transcription, mPTP opening, and mitochondrial potential $(\Delta \Psi \mathrm{m})$ detection}

The relative amounts of mitochondrial DNA (mtDNA) and nuclear DNA (nDNA) content were used to assess the mtDNA copy numbers via PCR [2]. The mtDNA and nuclear amplicons were generated from a complex IV segment and GAPDH segment, respectively. The transcript level of mtDNA was reflected by two different components: NADH dehydrogenase subunit 1 (ND1) and cytochrome c oxidase subunit I (COX I). The primers are described in the supplemental methods. Complex I, II, and IV activity was measured according to previous studies [46].

The opening of the mPTP was visualized as a rapid dissipation of tetramethylrhodamine ethyl ester fluorescence. Arbitrary mPTP opening time was determined as the time when tetramethylrhodamine ethyl ester fluorescence intensity decreased by half between initial and residual fluorescence intensity according to our previous study [2].

The $\Delta \Psi \mathrm{m}$ was analyzed using JC-1 staining (Beyotime Institute of Biotechnology). Briefly, cells were washed with ice-cold PBS and then stained with $2.5 \mathrm{~g} / \mathrm{ml} \mathrm{JC}-1$ for $30 \mathrm{~min}$ at $37^{\circ} \mathrm{C}$. After being washed with binding buffer, the cells were analyzed by fluorescence microscopy. Results are presented as relative aggregate-to-monomer (red/green) fluorescence intensity ratio.

\section{Statistical analysis}

Data analysis was conducted using SPSS 19.0 statistical software (SPSS, Chicago, IL, USA). Measurement data 
were presented as mean \pm SEM. The one-way analysis of variance was conducted among multi-groups. $P<0.05$ indicated statistical significance.

Funding This study was financially supported by grants from the National Natural Science Foundation of China (No. 81770237 and 81570225). The funders had no role in the study design, data collection and analysis, decision to publish, or preparation of the manuscript.

Author contributions H.Z. and Y.D.C. involved in conception and design, performance of experiments, data analysis and interpretation, and manuscript writing; J.W., P.J.Z., and H.Z. involved in the development of methodology, P.J.Z., H.Z., and J.R. involved in the data acquisition, H.Z. and P.J.Z. involved data analysis and interpretation; H.Z., Y.D.C., and J.R. involved in study supervision and final approval of manuscript.

\section{Compliance with Ethical Standards}

Conflict of Interest The authors declare that they have no conflict of interest.

Open Access This article is licensed under a Creative Commons Attribution 4.0 International License, which permits use, sharing, adaptation, distribution and reproduction in any medium or format, as long as you give appropriate credit to the original author(s) and the source, provide a link to the Creative Commons license, and indicate if changes were made. The images or other third party material in this article are included in the article's Creative Commons license, unless indicated otherwise in a credit line to the material. If material is not included in the article's Creative Commons license and your intended use is not permitted by statutory regulation or exceeds the permitted use, you will need to obtain permission directly from the copyright holder. To view a copy of this license, visit http://creativecommons. org/licenses/by/4.0/.

\section{References}

1. Buja LM. The pathobiology of acute coronary syndromes: clinical implications and central role of the mitochondria. Tex Heart Inst J. 2013;40:221-28.

2. Zhou H, Hu S, Jin Q, Shi C, Zhang Y, Zhu P, et al. Mff-dependent mitochondrial fission contributes to the pathogenesis of cardiac microvasculature ischemia/reperfusion injury via induction of mROS-mediated cardiolipin oxidation and HK2/VDAC1 disassociation-involved mPTP opening. J Am Heart Assoc. 2017;6:e05328.

3. Zhou H, Zhu P, Guo J, Hu N, Wang S, Li D, et al. Ripk3 induces mitochondrial apoptosis via inhibition of FUNDC1 mitophagy in cardiac IR injury. Redox Biol. 2017;13:498-507.

4. Anzell AR, Maizy R, Przyklenk K, Sanderson TH Mitochondrial quality control and disease: insights into ischemia-reperfusion injury. Mol Neurobiol. 2017.

5. Bravo-San Pedro JM, Kroemer G, Galluzzi L. Autophagy and mitophagy in cardiovascular disease. Circ Res. 2017;120: 1812-24.

6. Shi C, Cai Y, Li Y, Li Y, Hu N, Ma S, et al. Yap promotes hepatocellular carcinoma metastasis and mobilization via governing cofilin/F-actin/lamellipodium axis by regulation of JNK/Bnip3/SERCA/CaMKII pathways. Redox Biol. 2018;14: $59-71$.

7. Zhou H, Li D, Zhu P, Hu S, Hu N, Ma S, et al. Melatonin suppresses platelet activation and function against cardiac ischemia/reperfusion injury via PPARgamma/FUNDC1/mitophagy pathways. J Pineal Res. 2017;63:e12438.

8. Zhou H, Du W, Li Y, Shi C, Hu N, Ma S, et al. Effects of melatonin on fatty liver disease: The role of NR4A1/DNA-PKcs/ p53 pathway, mitochondrial fission, and mitophagy. J Pineal Res. 2018;64:e12450.

9. Jin Q, Li R, Hu N, Xin T, Zhu P, Hu S, et al. DUSP1 alleviates cardiac ischemia/reperfusion injury by suppressing the Mffrequired mitochondrial fission and Bnip3-related mitophagy via the JNK pathways. Redox Biol. 2018;14:576-87.

10. Gottlieb RA, Thomas A. Mitophagy and mitochondrial quality control mechanisms in the heart. Curr Pathobiol Rep. 2017;5:161-69.

11. Xiao B, Goh JY, Xiao L, Xian H, Lim KL, Liou YC. Reactive oxygen species trigger Parkin/PINK1 pathway-dependent mitophagy by inducing mitochondrial recruitment of Parkin. J Biol Chem. 2017;292:16697-708.

12. Georgakopoulos ND, Wells G, Campanella M. The pharmacological regulation of cellular mitophagy. Nat Chem Biol. 2017;13: 136-46.

13. Moulis MF, Millet AM, Daloyau M, Miquel MC, Ronsin B, Wissinger B, et al. OPA1 haploinsufficiency induces a BNIP3dependent decrease in mitophagy in neurons: relevance to Dominant Optic Atrophy. J Neurochem. 2017;140:485-94.

14. Zhou H, Zhang Y, Hu S, Shi C, Zhu P, Ma Q, et al. Melatonin protects cardiac microvasculature against ischemia/reperfusion injury via suppression of mitochondrial fission-VDAC1-HK2mPTP-mitophagy axis. J Pineal Res. 2017;63:e12413.

15. Feng Y, Madungwe NB, da Cruz Junho CV, Bopassa JC. Activation of $\mathrm{G}$ protein-coupled oestrogen receptor 1 at the onset of reperfusion protects the myocardium against ischemia/reperfusion injury by reducing mitochondrial dysfunction and mitophagy. Br J Pharmacol. 2017;174:4329-44.

16. Zhang W, Ren H, Xu C, Zhu C, Wu H, Liu D, et al. Hypoxic mitophagy regulates mitochondrial quality and platelet activation and determines severity of I/R heart injury. Elife. 2016;5:e21407.

17. Zhang W, Siraj S, Zhang R, Chen Q. Mitophagy receptor FUNDC1 regulates mitochondrial homeostasis and protects the heart from I/R injury. Autophagy. 2017;13:1080-81.

18. Kuang Y, Ma K, Zhou C, Ding P, Zhu Y, Chen Q, et al. Structural basis for the phosphorylation of FUNDC1 LIR as a molecular switch of mitophagy. Autophagy. 2016;12:2363-73.

19. Lv M, Wang C, Li F, Peng J, Wen B, Gong Q, et al. Structural insights into the recognition of phosphorylated FUNDC1 by LC3B in mitophagy. Protein Cell. 2017;8:25-38.

20. Munoz JP, Zorzano A. FUNDC1: A Novel Protein in Cardiac Health. Circulation. 2017;136:2267-70.

21. Battistutta R, Lolli G. Structural and functional determinants of protein kinase CK2alpha: facts and open questions. Mol Cell Biochem. 2011;356:67-73.

22. Chen G, Han Z, Feng D, Chen Y, Chen L, Wu H, et al. A regulatory signaling loop comprising the PGAM5 phosphatase and CK2 controls receptor-mediated mitophagy. Mol Cell. 2014;54: 362-77.

23. Bitirim CV, Tuncay E, Turan B Demonstration of subcellular migration of CK2alpha localization from nucleus to sarco(endo) plasmic reticulum in mammalian cardiomyocytes under hyperglycemia. Mol Cell Biochem. 2017.

24. Yang D, Wang T, Ni Y, Song B, Ning F, Hu P, et al. Apaminsensitive $\mathrm{K}+$ current upregulation in volume-overload heart failure is associated with the decreased interaction of CK2 with SK2. J Membr Biol. 2015;248:1181-89.

25. Hu N, Han X, Lane EK, Gao F, Zhang Y, Ren J. Cardiac-specific overexpression of metallothionein rescues against cigarette smoking exposure-induced myocardial contractile and mitochondrial damage. PLoS ONE. 2013;8:e57151. 
26. Ma Z, Xin Z, Di W, Yan X, Li X, Reiter RJ, et al. Melatonin and mitochondrial function during ischemia/reperfusion injury. Cell Mol Life Sci. 2017;74:3989-98.

27. Marchi S, Patergnani S, Missiroli S, Morciano G, Rimessi A, Wieckowski MR, et al. Mitochondrial and endoplasmic reticulum calcium homeostasis and cell death. Cell Calcium. 2017;69: 62-72.

28. Seldin DC, Lou DY, Toselli P, Landesman-Bollag E, Dominguez I. Gene targeting of CK2 catalytic subunits. Mol Cell Biochem. 2008;316:141-47.

29. Buchou T, Vernet M, Blond O, Jensen HH, Pointu H, Olsen BB, et al. Disruption of the regulatory beta subunit of protein kinase CK2 in mice leads to a cell-autonomous defect and early embryonic lethality. Mol Cell Biol. 2003;23:908-15.

30. Kim GS, Jung JE, Narasimhan P, Sakata H, Yoshioka H, Song YS, et al. Release of mitochondrial apoptogenic factors and cell death are mediated by CK2 and NADPH oxidase. J Cereb Blood Flow Metab. 2012;32:720-30.

31. Wang G, Ahmad KA, Ahmed K. Role of protein kinase CK2 in the regulation of tumor necrosis factor-related apoptosis inducing ligand-induced apoptosis in prostate cancer cells. Cancer Res. 2006;66:2242-49.

32. Sharp WW, Fang YH, Han M, Zhang HJ, Hong Z, Banathy A, et al. Dynamin-related protein 1 (Drp1)-mediated diastolic dysfunction in myocardial ischemia-reperfusion injury: therapeutic benefits of Drp1 inhibition to reduce mitochondrial fission. FASEB J. 2014;28:316-26.

33. Xie L, Liu S, Cheng J, Wang L, Liu J, Gong J. Exogenous administration of mitochondrial DNA promotes ischemia reperfusion injury via TLR9-p38 MAPK pathway. Regul Toxicol Pharmacol. 2017;89:148-54.

34. Reiter RJ, Mayo JC, Tan DX, Sainz RM, Alatorre-Jimenez M, Qin L. Melatonin as an antioxidant: under promises but over delivers. J Pineal Res. 2016;61:253-78.

35. Bartz RR, Suliman HB, Piantadosi CA. Redox mechanisms of cardiomyocyte mitochondrial protection. Front Physiol. 2015;6: 291.

36. Zhou H, Ma Q, Zhu P, Ren J, Reiter RJ, Chen Y. Protective role of melatonin in cardiac ischemia-reperfusion injury: from pathogenesis to targeted therapy. J Pineal Res. 2018;64:e12471.
37. Vasquez-Trincado C, Garcia-Carvajal I, Pennanen C, Parra V, Hill JA, Rothermel BA, et al. Mitochondrial dynamics, mitophagy and cardiovascular disease. J Physiol. 2016;594:509-25.

38. Liu L, Feng D, Chen G, Chen M, Zheng Q, Song P, et al. Mitochondrial outer-membrane protein FUNDC1 mediates hypoxia-induced mitophagy in mammalian cells. Nat Cell Biol. 2012;14:177-85.

39. Wu S, Lu Q, Wang Q, Ding Y, Ma Z, Mao X, et al. Binding of FUN14 domain containing 1 with inositol 1,4,5-trisphosphate receptor in mitochondria-associated endoplasmic reticulum membranes maintains mitochondrial dynamics and function in hearts in vivo. Circulation. 2017;136:2248-66.

40. Kravic B, Harbauer AB, Romanello V, Simeone L, Vogtle FN, Kaiser T, et al. In mammalian skeletal muscle, phosphorylation of TOMM22 by protein kinase CSNK2/CK2 controls mitophagy. Autophagy. 2017;1:1-25.

41. Diot A, Morten K, Poulton J. Mitophagy plays a central role in mitochondrial ageing. Mamm Genome. 2016;27:381-95.

42. Sin J, Andres AM, Taylor DJ, Weston T, Hiraumi Y, Stotland A, et al. Mitophagy is required for mitochondrial biogenesis and myogenic differentiation of $\mathrm{C} 2 \mathrm{C} 12$ myoblasts. Autophagy. 2016;12:369-80.

43. Rebholz H, Zhou M, Nairn AC, Greengard P, Flajolet M. Selective knockout of the casein kinase 2 in $\mathrm{d} 1$ medium spiny neurons controls dopaminergic function. Biol Psychiatry. 2013;74:113-21.

44. Zhou H, Yang J, Xin T, Zhang T, Hu S, Zhou S, et al. Exendin-4 enhances the migration of adipose-derived stem cells to neonatal rat ventricular cardiomyocyte-derived conditioned medium via the phosphoinositide 3-kinase/Akt-stromal cell-derived factor-1alpha/ CXC chemokine receptor 4 pathway. Mol Med Rep. 2015;11: 4063-72.

45. Zhou H, Yang J, Xin T, Li D, Guo J, Hu S, et al. Exendin-4 protects adipose-derived mesenchymal stem cells from apoptosis induced by hydrogen peroxide through the PI3K/Akt-Sfrp2 pathways. Free Radic Biol Med. 2014;77:363-75.

46. Janssen AJ, Trijbels FJ, Sengers RC, Smeitink JA, van den Heuvel LP, Wintjes LT, et al. Spectrophotometric assay for complex I of the respiratory chain in tissue samples and cultured fibroblasts. Clin Chem. 2007;53:729-34. 